Sir,

\section{Endogenous Pseudomonas endophthalmitis in an immunocompetent patient: a case for early diagnosis and treatment}

This report describes a case of Pseudomonas endogenous endophthalmitis in an otherwise healthy patient. The vision in the affected eye rapidly deteriorated and could not be restored. Investigations failed to reveal the source of primary infection.

\section{Case report}

A 57-year-old man presented to the eye department at a neighbouring hospital complaining of pain, blurring of vision and photophobia in his right eye of less than $24 \mathrm{~h}$ duration. He was otherwise fit and healthy.

On examination the visual acuity was counting fingers in the right eye, 6/5 in the left. Right-sided proptosis with restriction of eye movements and chemosis was noted. The intraocular pressure was elevated at $38 \mathrm{mmHg}$ and anterior uveitis was present. No hypopyon was detected and the fundus could not be visualised as the pupil failed to dilate. He was started on hourly ciprofloxacin and steroid drops. Systemic antibiotic treatment with ciprofloxacin was also added. Over the next $24 \mathrm{~h}$, as no improvement was noted, the systemic antibiotic treatment was changed to intravenous cefotaxime. FBC, ESR, blood glucose, ANA and rheumatoid factor were normal. His chest radiograph was normal while a radiograph of the paranasal sinuses was consistent with ethmoidal sinusitis. CT scan of the right orbit showed retro-orbital soft tissue thickening and inflammation involving the optic nerve and lateral rectus muscle. On the basis of these findings a diagnosis of orbital pseudotumour was suggested, although infection was not ruled out.

Systemic steroids were started. Over the next $48 \mathrm{~h}$, although the proptosis and the chemosis improved, the vision in the right eye dropped to PL. Fibrinous uveitis and vitritis was now present. The patient was then referred to this hospital.

On presentation to this department the patient had been symptomatic for over a week. The visual acuity in the right eye was PL with inaccurate projection, $6 / 5$ in the left. Chemosis, moderate proptosis and marked restriction of eye movements were present on the right. A relative afferent pupillary defect, raised intraocular pressure, fibrinous uveitis and a $1 \mathrm{~mm}$ hypopyon were noted. No view of the right fundus was obtained. A B-scan ultrasound was therefore done which confirmed a flat retina. A vitreous biopsy and anterior chamber paracentesis was performed and intravitreal amikacin and vancomycin administered. Gram-negative bacilli, subsequently confirmed as Pseudomonas aeruginosa sensitive to amikacin and ciprofloxacin, were cultured. The orbital cellulitis resolved but the vision dropped further to NPL over a week despite repeated intravitreal amikacin.
A complete septic screen was carried out to trace the source of infection. A repeat FBC showed mild leucocytosis. ESR, U\&E, chest radiograph and a radiograph of the paranasal sinuses were also repeated and were normal. Blood, mid-stream urine, stool and sputum microscopy and cultures were all negative. Abdominal ultrasound and cardiac ultrasound failed to reveal the source of infection.

\section{Comment}

Endogenous endophthalmitis is usually seen as a sequel of septicaemia, endocarditis, meningitis or intraabdominal infection. The organisms commonly implicated in endogenous endophthalmitis include Streptococcus, ${ }^{1}$ Pseudomonas, ${ }^{2}$ Listeria $^{3,4}$ and Fusarium. ${ }^{5}$ Aspergillus, ${ }^{6}$ Rickettsia, Candida ${ }^{7}$ and Cryptococcus $^{8}$ have also been noted to cause endogenous endophthalmitis in the immunocompromised host. Endogenous endophthalmitis in the immunocompetent host, although a rare entity, has been described. The organisms isolated in such a setting include Streptococcus mitis, ${ }^{1}$ Listeria monocytogenes, ${ }^{3,4}$ Fusarium solani ${ }^{5}$ and Aspergillus fumigatus. ${ }^{9}$ To our knowledge this is the first case of Pseudomonas endogenous endophthalmitis described in an immunocompetent host.

The visual outcome in such cases is directly related to the virulence of the organism and the immunocompetence of the host. In general, endophthalmitis caused by Gram-negative organisms such as Pseudomonas aeruginosa, Haemophilus influenzae, Proteus species, Klebsiella pneumoniae, Enterobacter species and Escherichia coli have a poor visual prognosis. ${ }^{10}$ In a study of 52 patients with culture-proven Gram-negative endophthalmitis, Irvine et al. ${ }^{10}$ found Pseudomonas aeruginosa was associated with the worst visual prognosis. Less than $10 \%$ of patients infected with this organism attained a final visual acuity of $20 / 400$. Other reports ${ }^{11-13}$ have shown that in $70-80 \%$ of such cases the visual outcome is a blind eye. Important factors associated with a poor visual outcome in endophthalmitis are delayed or inadequate treatment, positive cultures, poor initial visual acuity and accidental trauma. ${ }^{10}$ The first three of these adverse prognostic factors were seen in our case.

This patient also demonstrated severe orbital cellulitis with proptosis and restriction of ocular movements in addition to the endophthalmitis. As pointed out by McLeod et al., ${ }^{14}$ the cellulitis appears to be a response to the virulence of the infecting organism. Whether the orbital cellulitis was an inflammatory response to the endophthalmitis or a result of direct involvement by Pseudomonas is uncertain.

A critical factor responsible for the poor outcome in this case was the initial management at the referring department, which was less than ideal. The correct diagnosis of endophthalmitis was not made until the patient was seen at the referral hospital, by which time it was too late to save vision. The initial management of the patient was with topical ciprofloxacin and steroid drops 
followed by systemic antibiotics and steroids. Intravitreal antibiotics were given only a week after the onset of his symptoms, on admission to the referral hospital.

The rapid loss of vision despite subsequent aggressive intraocular treatment with antibiotics, bears testimony to the pathogenicity of Pseudomonas endophthalmitis. This case illustrates once again that intravenous antibiotics are the wrong treatment for acute infectious endophthalmitis even where the organism is sensitive to the antibiotic.

The mainstay of bacterial endophthalmitis treatment is intravitreal antibiotics. The antibiotics used for intravitreal injection include amikacin $(0.4 \mathrm{mg}$ in $0.1 \mathrm{ml})$, vancomycin (1 mg in $0.1 \mathrm{ml})$, cefuroxime (1 mg in $0.1 \mathrm{ml})$, gentamicin $(0.2 \mathrm{mg}$ in $0.1 \mathrm{ml})$, and clindamycin $(1 \mathrm{mg}$ in $0.1 \mathrm{ml}) .{ }^{15}$ Vancomycin is considered the drug of choice for Gram-positive infection and acts by inhibiting the synthesis of peptidoglycan, a major component of the bacterial cell wall. Aminoglycosides such as amikacin and gentamicin which inhibit intracellular protein synthesis are used in the management of Gram-negative infections. Ceftazidime is also a useful alternative against Gram-negative organisms and acts by inhibiting the transpeptidase reaction which cross links the bacterial cell wall. ${ }^{16}$ The Endophthalmitis Virectomy Study, ${ }^{17}$ which looked at the management of post-operative endophthalmitis, showed there was no advantage in the concurrent administration of topical antibiotics unless there are specific problems such as microbial keratitis or wound infection. The study also established that addition of systemic antibiotics did not confer any advantage in post-operative endophthalmitis. However, in the management of endogenous endophthalmitis, especially in cases with proven bacteremia, systemic antibiotics have been used as an adjunct to intravitreal antibiotics. ${ }^{18}$ While exogenous endophthalmitis and endogenous endophthalmitis in the immunocompromised host are well-recognised entities, a high index of suspicion is vital in diagnosing endogenous endophthalmitis in the immunocompetent patient. This case once again underlines the need for early diagnosis and administration of intravitreal antibiotics in the management of endophthalmitis.

\section{References}

1. Harrison SA, Bateman JB. Endogenous endophthalmitis caused by Streptococcus mitis. Am J Ophthalmol 1997;123:260-1

2. Mu SC, Lin CH, Lin MI, Hu CC. Pseudomonas aeruginosa endophthalmitis in prematurity: report of two cases. ChungHua Min Kuo Hsiao Erh Ko I Hsueh Hui Tsa Chih 1997;38:159-61.

3. Lohmann CP, Heep M, Linde HJ, Reischl U. Listeria monocytogenes-induced endogenous endophthalmitis in an otherwise healthy patient: PCR-assisted rapid diagnosis as the basis for successful therapy. Klin Monatsbl Augenheilkd 1998;212:55-8.

4. Eliott D, O'Brien TP, Green WR, Jampel HD, Goldberg MF. Elevated intraocular pressure, pigment dispersion and dark hypopyon in endogenous endophthalmitis from Listeria monocytogenes. Surv Ophthalmol 1992;37:117-24.
5. Louie T, el Baba F, Shulman M, Jimenez-Loucho V. Endogenous endophthalmitis due to Fusarium: case report and review. Clini Infect Dis 1994;18:585-8.

6. Weishaar PD, et al. Endogenous Aspergillis endophthalmitis. Ophthalmology 1998;105:57-65.

7. Chen S, Chung Y, Liu J. Endogenous Candida endophthalmitis after induced abortion. Am J Ophthalmol 1998;125:873-5.

8. Sheu S, Chen Y, Kuo N, Wang J, Chen C. Endogenous cryptococcal endophthalmitis. Am J Ophthalmol 1998;105:377-81.

9. Velluri S, Moorthy RS, Ligett PE, Rao NA. Endogenous Aspergillus endophthalmitis in an immunocompetent host. Int Ophthalmol 1993;17:131-5.

10. Irvine WD, Flynn HW, Miller D, Pflugfelder SC. Endophthalmitis caused by gram negative organisms. Arch Ophthalmol 1992;110:1450-4.

11. Bohigian GM, Olk RJ. Factors associated with a poor visual result in endophthalmitis. Am J Ophthalmol 1986;101:332-4.

12. Puliafito CA, Baker AS, Haaf J, Foster CS. Infectious endophthalmitis: a review of 36 cases. Ophthalmology 1982;89:921-9.

13. Mandelbaum S, Forster RK, Gelender H, Culbertson W. Late onset endophthalmitis associated with filtering blebs. Ophthalmology 1985;92:964-72.

14. McLeod SD, Flowers CW, Lopez PF, Marx J, McDonell PJ. Endophthalmitis and orbital cellulitis after radial keratotomy. Ophthalmology 1995;102:1902-7.

15. Update from the Royal College of Ophthalmolgists. Management of endophthalmitis. Focus 1996;1:1-2.

16. Roth DB, Flynn HW JR. Antibiotic selection in the treatment of endophthalmitis: the significance of drug combinations and synergy. Surv Ophthalmol 1997;41:395-401.

17. The Endophthalmitis Vitrectomy Study Group. Results of the Endophthalmitis Vitrectomy Study. Arch Ophthalmol 1995;113:1479-96.

18. Okada AA, Johnson RP, Liles WC, D'Amico DJ, Baker AS. Endogenous bacterial endophthalmitis: report of a ten year retrospective study. Ophthalmology 1994;101:832-8.

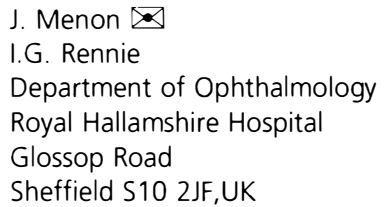

Sir,

\section{Cortical blindness following pre-eclampsia}

Pre-eclampsia is a disease characterised by hypertension, proteinuria and oedema in late pregnancy. It occurs in as many as $10 \%$ of all pregnancies and is a major cause of maternal and fetal morbidity. ${ }^{1}$ It is a multisystem disorder that affects hepatic, renal and coagulation systems. Until its terminal phase it is asymptomatic but can rapidly proceed to generalised seizures (eclampsia).

Visual disturbances are reported in $20 \%$ of women with pre-eclampsia and in as many as $50 \%$ with eclampsia. ${ }^{2}$ Isolated transient cortical blindness is rare in pre-eclampsia, reportedly occurring in $1-3 \%$ of cases.,

We describe a unique case of isolated cortical blindness in a woman with pre-eclampsia resulting in a degree of permanent visual loss. Typical occipital and parietal lobe cerebral oedema were seen on computed tomography $(\mathrm{CT})$ and magnetic resonance imaging (MRI). 